\title{
Near future MeV telescopes can discover asteroid-mass primordial black hole dark matter
}

\author{
Anupam Ray $\odot$, ${ }^{, *}$ Ranjan Laha ${ }^{2, \dagger}$ Julian B. Muñoz $\odot,{ }^{3, \ddagger}$ and Regina Caputo $\odot^{4, \S}$ \\ ${ }^{1}$ Tata Institute of Fundamental Research, Homi Bhabha Road, Mumbai 400005, India \\ ${ }^{2}$ Centre for High Energy Physics, Indian Institute of Science, \\ C.V.Raman Avenue, Bengaluru 560012, India \\ ${ }^{3}$ Harvard-Smithsonian Center for Astrophysics, 60 Garden St., Cambridge, Massachusetts 02138, USA \\ ${ }^{4}$ NASA Goddard Space Flight Center, Greenbelt, Maryland 20771, USA
}

(Received 23 February 2021; accepted 9 June 2021; published 15 July 2021)

\begin{abstract}
Primordial black holes (PBHs), formed out of large overdensities in the early Universe, are a viable dark matter (DM) candidate over a broad range of masses. Ultralight, asteroid-mass PBHs with masses around $10^{17} \mathrm{~g}$ are particularly interesting as current observations allow them to constitute the entire DM density. PBHs in this mass range emit $\sim \mathrm{MeV}$ photons via Hawking radiation which can directly be detected by the gamma ray telescopes, such as the upcoming AMEGO. In this work we forecast how well an instrument with the sensitivity of AMEGO will be able to detect, or rule out, PBHs as a DM candidate, by searching for their evaporating signature when marginalizing over the Galactic and extra-Galactic gamma-ray backgrounds. We find that an instrument with the sensitivity of AMEGO could exclude nonrotating PBHs as the only DM component for masses up to $7 \times 10^{17} \mathrm{~g}$ at $95 \%$ confidence level for a monochromatic mass distribution, improving upon current bounds by nearly an order of magnitude. The forecasted constraints are more stringent for $\mathrm{PBHs}$ that have rotation, or which follow extended mass distributions.
\end{abstract}

DOI: $10.1103 /$ PhysRevD.104.023516

\section{INTRODUCTION}

Unequivocal evidence of a nonbaryonic form of matter, known as dark matter (DM), as a dominant component of the Universe has been confirmed by numerous astrophysical and cosmological observations [1-3]. Experimental searches for the elusive DM have thus far shown no firmly preferred model [4-6]. Primordial black holes (PBHs), possibly formed via gravitational collapse of large overdensities in the early universe or via other exotic mechanisms, are one of the earliest proposed and well-motivated DM candidates [7-10]. PBHs have a wide range of masses and can constitute a large fraction or even the entirety of the DM density [11-17]. The idea of PBH DM has recently received renewed attention with the first detection of a $\mathrm{BH}$ merger by the LIGO-Virgo Collaboration [18], argued to have a PBH rather than astrophysical origin [19-21]. Several techniques have been implemented to probe the DM fraction of PBHs over a wide mass range. These have resulted in a multitude of observational constraints [13,14,22-57], along with several future projections $[11,29,58-72]$ along a broad range of PBH masses.

\footnotetext{
*anupam.ray@theory.tifr.res.in ranjanlaha@iisc.ac.in

\#ulianmunoz@cfa.harvard.edu

regina.caputo@nasa.gov
}

Due to their Hawking emission, extremely light PBHs would have evaporated by today, setting a lower limit on the mass of $\sim 5 \times 10^{14} \mathrm{~g}$ for nonrotating PBHs (or $\sim 7 \times$ $10^{14} \mathrm{~g}$ if maximally rotating) [73-75]. PBHs heavier than that still evaporate, and act as decaying DM. Ultralight PBHs with masses in between $5 \times 10^{14} \mathrm{~g}-2 \times 10^{17} \mathrm{~g}$, are typically probed via searches of their Hawking radiation. Non-observations of such Hawking-produced photons [22,29,30,56], neutrinos [14], and electrons/positrons [14,26-28,76-79] provide the leading constraints on ultralight PBHs. Additional constraints in this mass range are also obtained via precise observations of the cosmic microwave background and big bang nucleosynthesis [23,80-82]. PBHs in the mass range of $\sim 2 \times 10^{17} \mathrm{~g}-10^{23} \mathrm{~g}$, often known as the asteroid-mass range [83], are currently allowed to compose the entirety of the DM [11-13]. Unlike solar-mass BHs, these ultralight $\mathrm{BHs}$ cannot be produced by any known astrophysical processes (even with the continued accumulation of asymmetric DM particles in compact objects $[84,85])$, and thus would be a smoking gun of new physics, be it during the early Universe or in a complex dark sector [86].

In this work, we propose a technique to decisively probe a part of the parameter space for PBH DM in the asteroidmass range. We show that observation of the Galactic Center by future $\mathrm{MeV}$ telescopes, such as an instrument with the sensitivity of AMEGO [87], can probe the DM fraction of asteroid-mass PBHs. AMEGO can exclude 
nonrotating (maximally rotating) $\mathrm{PBHs}$ as the sole component of DM upto $\sim 7 \times 10^{17} \mathrm{~g}\left(\sim 4 \times 10^{18} \mathrm{~g}\right)$, at $95 \%$ confidence level (C.L.), assuming no signal is present in the data and a monochromatic mass function of PBHs. Assuming that PBHs follow an extended mass distribution (log-normal distribution with width $\sigma=0.5$ ), AMEGO can probe further into an entirely unexplored mass window, improving our current constraints by nearly an order of magnitude and pushing us closer to probe the entire asteroid-mass PBH window. Ref. [56], which appeared as our paper was near completion, performs a similar study for nonrotating PBHs with a monochromatic mass distribution. Our work differs from Ref. [56] in several key aspects (e.g., the inclusion of the extra-Galactic astrophysical background, and a different region of interest in the Galactic Center), chief among them is our usage of Fisher analysis to derive the projected exclusion limits on the DM fraction of ultralight PBHs including marginalization over the astrophysical parameters.

\section{PARTICLE EMISSION FROM EVAPORATING BLACK HOLES}

BHs evaporate via Hawking radiation [8]. An uncharged and rotating $\mathrm{BH}$ of mass $M_{\mathrm{BH}}$ and angular momentum $J_{\mathrm{BH}}$ radiates at a temperature [73-75,88,89]

$$
T_{\mathrm{BH}}=\frac{1}{4 \pi G_{N} M_{\mathrm{BH}}} \frac{\sqrt{1-a_{*}^{2}}}{1+\sqrt{1-a_{*}^{2}}},
$$

where $G_{N}$ denotes the gravitational constant and $a_{*}=$ $J_{\mathrm{BH}} /\left(G_{N} M_{\mathrm{BH}}^{2}\right)$ is the dimensionless spin parameter. For a given $\mathrm{BH}$ mass, the temperature can vary by orders of magnitude as it approaches its maximal spin, $a_{*} \rightarrow 1$, where the BH stops evaporating.

The number of emitted particles from an evaporating $\mathrm{BH}$ of mass $M_{\mathrm{BH}}$ and dimensionless spin parameter $a_{*}$, in the energy interval $E$ and $E+d E$ and in a time interval $d t$ follows a blackbody like distribution $[8,73-75,88,89]$

$$
\frac{d^{2} N}{d E d t}=\frac{1}{2 \pi} \frac{\Gamma_{s}\left(E, M_{\mathrm{BH}}, a_{*}, \mu\right)}{\exp \left[E^{\prime} / T_{\mathrm{BH}}\right]-(-1)^{2 s}},
$$

where $E^{\prime}$ denotes the effective energy of the emitted particles including the rotational velocity of the $\mathrm{BH} . \Gamma_{s}$ denotes the graybody factor which accounts for the departure from an ideal blackbody emission. It depends on the spin of the emitted particle $s$, rest mass of the emitted particle $\mu$, and the BH mass and spin. In the high energy limit, $G_{N} M_{\mathrm{BH}} E \gg 1$, the graybody factor becomes independent of the spin of the emitted particle species and reaches its geometric saturation value, i.e., $\Gamma_{s}=27 G_{N}^{2} M_{\mathrm{BH}}^{2} E^{2}$. In the opposite limit, $G_{N} M_{\mathrm{BH}} E \ll 1$, it strongly depends on the spin of the emitted particle species $[73,88]$.
For this work, we use the publicly available BlackHawk [90] package to generate the emitted particle spectrum from evaporating BHs. We have verified this numerically obtained emission rate against semianalytical formulas from Ref. [73,74,88].

\section{METHODS AND RESULTS}

Hereafter we focus on primordial black holes. Ultralight PBHs emit significant number of photons of energy comparable to their temperature. More precisely, photon emission peaks at an energy $E \sim 5.77 T_{\mathrm{PBH}}[75,88]$ for an evaporating $\mathrm{PBH}$ with temperature $T_{\mathrm{PBH}}$. The emission of photons is exponentially suppressed for energies exceeding $T_{\mathrm{PBH}}\left(E \gg T_{\mathrm{PBH}}\right)$, and falls off as a power law in the opposite limit $\left(E \ll T_{\mathrm{PBH}}\right)$.

For a monochromatic mass distribution of $\mathrm{PBHs}$, the Galactic contribution to the differential flux from $\mathrm{PBH}$ evaporation is

$\left.\frac{d \phi_{\mathrm{gal}}}{d E}\right|_{\mathrm{mono}}=\frac{f_{\mathrm{PBH}}}{4 \pi M_{\mathrm{PBH}}} \frac{d^{2} N}{d E d t} \int_{0}^{s_{\max }} \rho[r(s, l, b)] d s d \Omega$,

where $f_{\mathrm{PBH}}$ denotes the DM fraction of PBHs. DM profile of the Milky Way (MW) is denoted by $\rho[r(s, l, b)]$, where $r$ is the Galacto-centric distance, $s$ is the distance from the observer, $l$ and $b$ denote the Galactic longitude and latitude respectively, and $d \Omega=\cos [b] d l d b$ is the differential solid angle under consideration. The upper limit of the line of sight integral, $s_{\max }$, depends on the size of the MW DM halo, Galactic longitude, and Galactic latitude,

$s_{\max }=r_{\odot} \cos [b] \cos [l]+\sqrt{r_{\max }^{2}-r_{\odot}^{2}\left(1-\cos ^{2}[b] \cos ^{2}[l]\right)}$,

where $r_{\max }$ denotes the maximum size of the MW halo, and $r_{\odot}$ is the Galacto-centric distance of the Sun.

The extra-Galactic contribution to the differential flux for a monochromatic mass distribution of PBHs is

$\left.\frac{d \phi_{\mathrm{eg}}}{d E}\right|_{\text {mono }}=\left.\frac{\Delta \Omega}{4 \pi} \frac{f_{\mathrm{PBH}} \rho_{\mathrm{DM}}}{M_{\mathrm{PBH}}} \int_{z=0}^{\infty} \frac{d z}{H(z)} \frac{d^{2} N}{d E d t}\right|_{E \rightarrow[1+z] E}$,

where $\Delta \Omega$ denotes the total solid angle under consideration, $\rho_{\text {DM }}$ is the average DM density of the Universe at the present epoch, and $H(z)=H_{0} \sqrt{\Omega_{\Lambda}+\Omega_{m}(1+z)^{3}+\Omega_{r}(1+z)^{4}}$ is the Hubble expansion rate at redshift $z$. The Hubble expansion rate at the present epoch is $H_{0} ; \Omega_{\Lambda}, \Omega_{m}$, and $\Omega_{r}$ denote the current dark-energy, matter, and radiation densities of the Universe, respectively. For numerical values of all cosmological parameters, we use the latest Planck 2018 measurements [1].

In addition to a monochromatic mass distribution for PBHs, we also consider a log-normal mass distribution, as predicted by various inflationary models 


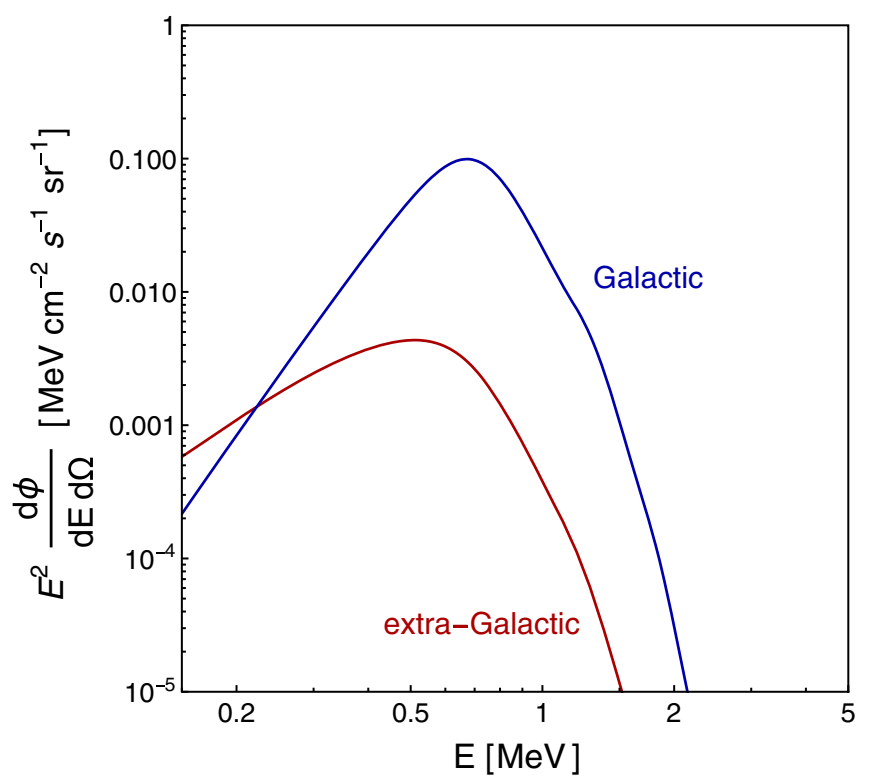

FIG. 1. The Galactic and extra-Galactic photon contributions from Hawking evaporation off a nonrotating PBHs of mass $10^{17} \mathrm{~g}$. It is assumed that PBHs make up the entirety of DM and follow an NFW density profile. The blue and red lines correspond to the Galactic and extra-Galactic contributions in the region of interest $(|l| \leq 5 \mathrm{deg}$ and $|b| \leq 5 \mathrm{deg})$ respectively.

$$
\frac{d N_{\mathrm{PBH}}}{d M_{\mathrm{PBH}}}=\frac{1}{\sqrt{2 \pi} \sigma M_{\mathrm{PBH}}} \exp \left[-\frac{\ln ^{2}\left(M_{\mathrm{PBH}} / \mu_{\mathrm{PBH}}\right)}{2 \sigma^{2}}\right],
$$

where $\mu_{\mathrm{PBH}}$ and $\sigma$ are the mean mass and width of the distribution. For an extended mass distribution of PBHs, the (extra-)Galactic contribution to the differential flux is

$$
\left.\frac{d \phi_{\mathrm{gal}, \mathrm{eg}}}{d E}\right|_{\mathrm{ext}}=\left.\int d M_{\mathrm{PBH}} \frac{d N_{\mathrm{PBH}}}{d M_{\mathrm{PBH}}} \frac{d \phi_{\mathrm{gal}, \mathrm{eg}}}{d E}\right|_{\mathrm{mono}} .
$$

For nonrotating $\mathrm{PBHs}$, the mass integral runs from $M_{\min }=5 \times 10^{14} \mathrm{~g}$ to $M_{\max }=\infty$. For PBHs approaching to their maximal rotation, the mass integral runs from $M_{\min }=7 \times 10^{14} \mathrm{~g}$ to $M_{\max }=\infty$, as the maximal rotation increases the minimum evaporation mass $M_{\min }$ by enhancing the Hawking emission rate. Note that, the minimum PBH mass only matters for extended PBH distributions with low averages.

Fig. 1 shows the Galactic and extra-Galactic contributions to the total evaporation flux from PBHs of mass $10^{17} \mathrm{~g}$ in Galactic Center, defined to have: $|l| \leq 5 \mathrm{deg}$ and $|b| \leq 5 \mathrm{deg}$. Since this region of interest resides in a DMdominated environment, the extra-Galactic contribution to the evaporation signal is always subdominant. Galactic emission peaks at around $\sim 0.6 \mathrm{MeV}$ as the temperature of a $10^{17} \mathrm{~g} \mathrm{PBH}$ is $0.1 \mathrm{MeV}$. The extra-Galactic signal peaks at a slightly lower energy as it is redshifted.

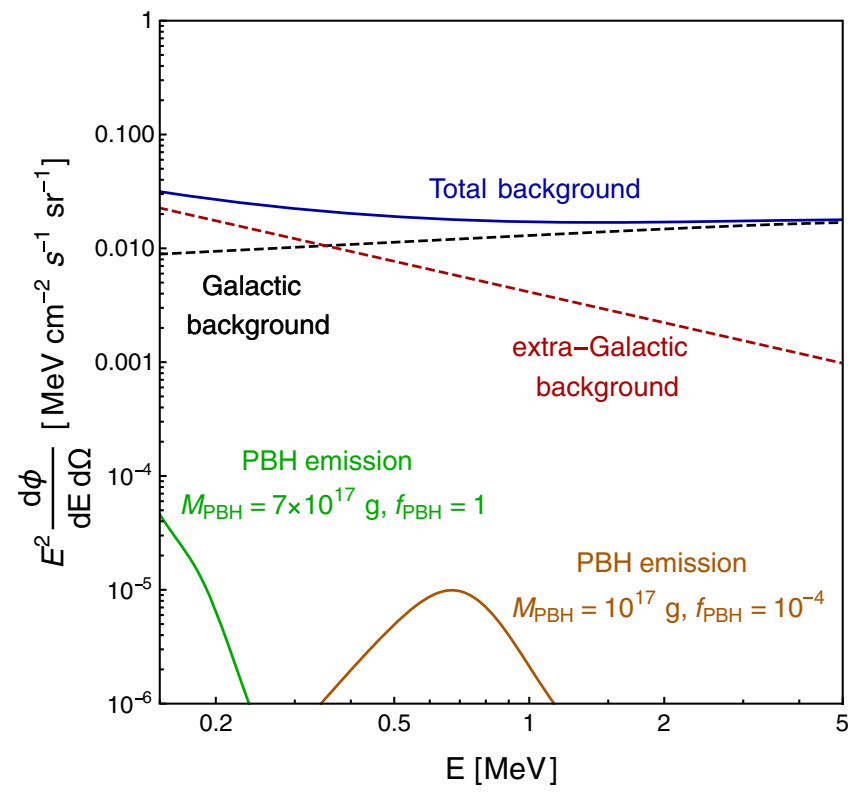

FIG. 2. Galactic and extra-Galactic astrophysical backgrounds are shown as a function of the emitted photon energy. Dashed black line corresponds to the Galactic background which is adapted from Ref. [91]. Dashed red line corresponds to the extraGalactic background which is a single power law fit to the Cosmic X-ray background measurements. Total background, sum of the Galactic and extra-Galactic backgrounds, is shown by the solid blue line. Evaporation signals from nonrotating PBHs of mass $10^{17} \mathrm{~g}$ with dark matter fraction of $10^{-4}$ and a nonrotating $\mathrm{PBH}$ of mass $7 \times 10^{17} \mathrm{~g}$ with dark matter fraction of unity are shown for comparison.

Of course, PBHs are not the only possible source of gamma rays in the cosmos. In particular, there are well-known astrophysical backgrounds, which we ought to marginalize over to unearth a possible PBH signal. Fig. 2 shows the Galactic and extra-Galactic astrophysical backgrounds used in this analysis. We have adapted the Galactic astrophysical background $\phi_{\text {gal }}^{\mathrm{bkg}}$ from Ref. [91],

$$
\phi_{\mathrm{gal}}^{\mathrm{bkg}}(E)=A_{\mathrm{bkg}}\left(\frac{E}{1 \mathrm{MeV}}\right)^{-\alpha} \exp \left[-\left(\frac{E}{E_{c}}\right)^{\gamma}\right] \text {, }
$$

in units of $\mathrm{MeV}^{-1} \mathrm{~cm}^{-2} \mathrm{~s}^{-1} \mathrm{sr}^{-1}$, which contains four parameters: an amplitude $\left(A_{\mathrm{bkg}}\right)$, power law index $(\alpha)$, exponential cut off energy $\left(E_{c}\right)$, and the index within the exponential $(\gamma)$. Their best fit values are $A_{\mathrm{bkg}}=0.013 \mathrm{MeV}^{-1} \mathrm{~cm}^{-2} \mathrm{~s}^{-1} \mathrm{sr}^{-1}$, $\alpha=1.8, E_{c}=20 \mathrm{MeV}$, and $\gamma=2$, respectively. We have checked that this formula provides an adequate fit to the data obtained by COMPTEL [92-94]. For the extra-Galactic background, $\phi_{\mathrm{eg}}^{\mathrm{bkg}}$, we have considered a single power law which fits the cosmic $\mathrm{x}$-ray background spectrum measured by various experiments [95-99] in the energy range $150 \mathrm{keV}$ to $5 \mathrm{MeV}$ [29], 


$$
\phi_{\mathrm{eg}}^{\mathrm{bkg}}(E)=A_{\mathrm{bkg}}^{\mathrm{eg}}\left(\frac{E}{1 \mathrm{MeV}}\right)^{-\alpha^{\mathrm{eg}}}
$$

also in $\mathrm{MeV}^{-1} \mathrm{~cm}^{-2} \mathrm{~s}^{-1} \mathrm{sr}^{-1}$. Our power-law model for the extra-Galactic background contains two parameters, its amplitude $\left(A_{\mathrm{bkg}}^{\mathrm{eg}}\right)$, and the power law index $\left(\alpha^{\mathrm{eg}}\right)$, with best-fit values of $A_{\mathrm{bkg}}^{\mathrm{eg}}=0.004135 \mathrm{MeV}^{-1} \mathrm{~cm}^{-2} \mathrm{~s}^{-1} \mathrm{sr}^{-1}$, and $\alpha^{\mathrm{eg}}=2.8956$.

We consider photons in the energy range $0.15-5 \mathrm{MeV}$ for this analysis. The lower end of the energy range is determined by the sensitivity of AMEGO, whereas, the higher end of the energy range is determined by the evaporation signal. For PBHs of mass $2 \times 10^{16} \mathrm{~g}$ (minimum mass considered for this analysis), evaporation signal peaks at around $3 \mathrm{MeV}$, and falls off exponentially with increase in photon energy. Moreover, the single power-law fit to the extra-Galactic background in Eq. (9) is valid only up to $\sim 5 \mathrm{MeV}$ [29].

We have applied Fisher forecasting [100-102] with marginalization over all astrophysical background parameters to compute the projected upper limits at 95\% C.L. The exclusion limits are derived by assuming no evaporation signal is present in the data. The Fisher information matrix $(\mathcal{F})$ is a $N \times N$ matrix, where $N$ denotes the total number of parameters $\vec{p}=\left\{p_{1}, p_{2}, \ldots, p_{N}\right\}$ and is defined as [91]

$\mathcal{F}_{i j}=\int_{E} \int_{\Omega} \frac{\partial_{i} \phi(E, \Omega) \partial_{j} \phi(E, \Omega)}{\phi(E, \Omega)} T_{\mathrm{obs}} A_{\mathrm{eff}}(E) d \Omega d E$,

where $\phi(E, \Omega)=\left(\phi_{\mathrm{gal}}+\phi_{\mathrm{eg}}+\phi_{\mathrm{gal}}^{\mathrm{bkg}}+\phi_{\mathrm{eg}}^{\mathrm{bkg}}\right)$ is the total flux, $T_{\text {obs }}$ is the observation time, and $A_{\text {eff }}(E)$ is the effective area. We conservatively ignore the extraGalactic PBH emission, as it is subdominant in our region of interest. The effective area for AMEGO is adapted from Ref. [87,103] and a uniform sky coverage $T_{\text {obs }}$ of one year is considered for this analysis. We use a sufficiently dense binning in order to capture all the spectral variations in the Fisher information matrix. We have considered 2000 logarithmically spaced bins between the energy interval of $0.15-5 \mathrm{MeV}$.

For this work, there is only one signal parameter, the fraction $f_{\mathrm{PBH}}$ of the $\mathrm{DM}$ that is composed of $\mathrm{PBHs}$ for each mass we study, plus the six astrophysical parameters introduced in Eqs. (8) and (9). Hence, the Fisher information matrix $(\mathcal{F})$ used in our analysis is a $7 \times 7$ symmetric matrix. The projected upper limit on the signal parameter $f_{\mathrm{PBH}}$ at $95 \%$ C.L. is [91]

$$
f_{\mathrm{PBH}}^{\mathrm{UL}}=1.645 \sqrt{\left(\mathcal{F}^{-1}\right)_{11}} \text {. }
$$

Because of the relatively large region of interest, our results are almost insensitive to different choices of DM density profiles. For this work, we assume that the density

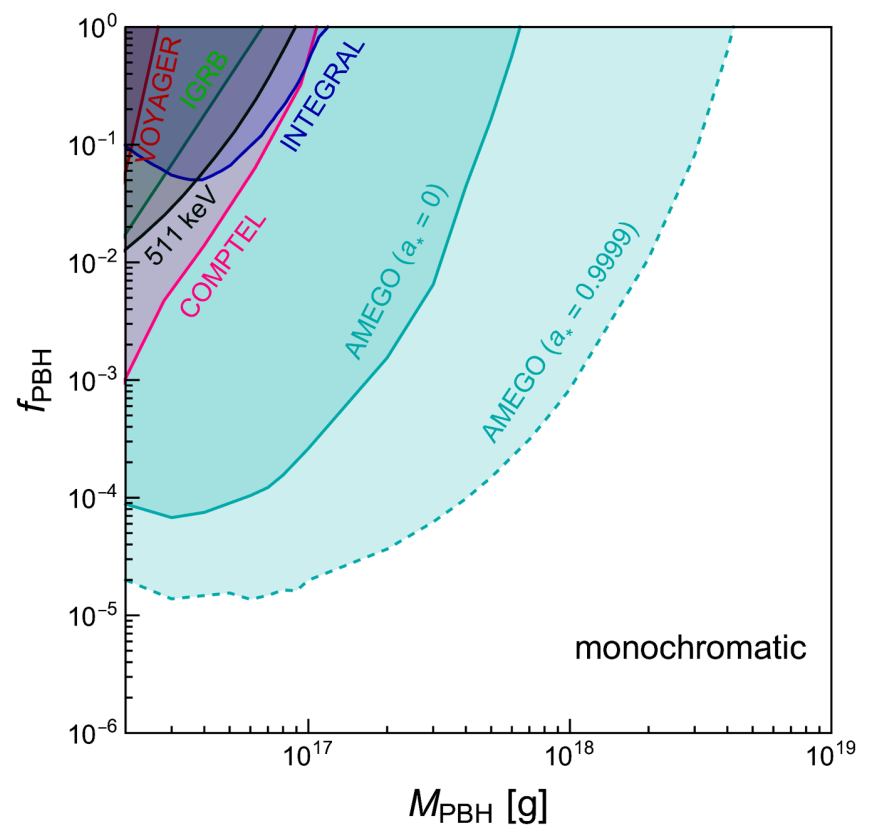

FIG. 3. Projected upper limit (95\% C.L.) on the DM fraction of PBHs, $f_{\mathrm{PBH}}$, from near future $\mathrm{MeV}$ telescope AMEGO. The plot corresponds to a monochromatic mass distribution of PBHs. Results for nonrotating PBHs $\left(a_{*}=0\right)$ and PBHs approaching to their maximal spin $\left(a_{*}=0.9999\right)$ are shown by the solid line and the dashed line respectively. The constraints are derived by considering an NFW density profile of the ultralight PBHs. The existing exclusions on ultralight nonspinning $\mathrm{PBHs}$ from Voyager-1 measurement of positron flux (shaded red) [26], extraGalactic gamma ray emission(shaded green) [22,29,109], SPI/ INTEGRAL $511 \mathrm{keV}$ emission line with $1.5 \mathrm{kpc}$ positron annihilation region and isothermal DM profile (shaded black) $[14,27,28]$ and INTEGRAL, COMPTEL Galactic Center MeV flux (shaded blue, shaded magenta) $[30,56]$ are also shown for comparison. For reference, there are no existing exclusion limits to the right of the plot until $M_{\mathrm{PBH}} \sim 10^{23} \mathrm{~g}[11-13,110]$.

distribution of ultralight PBHs in MW halo follows a Navarro-Frenk-White (NFW) profile [104]. However, we have tested our results with other density profiles such as with a cored isothermal profile [105] and with a cored NFW profile with a core radii of $2 \mathrm{kpc}$ [30]. We find that due to the different choices of DM density profiles, our results alter by as far as a factor of two (degrades by a factor of $\sim 1.69$ for a cored isothermal profile and by a factor of $\sim 1.55$ for a cored NFW profile with a core radius of $2 \mathrm{kpc}$ ). We have also checked that a somewhat larger region of interest around the Galactic Center, say $|l| \leq 30 \mathrm{deg}$ and $|b| \leq 10 \mathrm{deg}$, increases the Hawking evaporated photons as well as background photons by a factor of 6.81 and 12 respectively, indicating a putative improvement of the projections by a factor of $(S / \sqrt{N}) \sim 1.96$ (which, however, may be reduced by marginalization with the Fisher matrix).

Fig. 3 shows the projected upper limits (at 95\% C.L.) on $\mathrm{DM}$ fraction of $\mathrm{PBHs}, f_{\mathrm{PBH}}$, that can be derived from future 
AMEGO observations by assuming that no DM signal is present in the data. Monochromatic mass distribution of the PBHs is assumed in this plot. The solid/dashed lines correspond to nonrotating $\left(a_{*}=0\right)$ /approaching maximal rotation $\left(a_{*}=0.9999\right)$ PBHs. Note that, we take the maximum value of spin as 0.9999 because BlackHawk can not go beyond that. The limits are derived by assuming an NFW density profile of the PBHs. As the PBHs become maximally rotating, their temperatures as well as the effective energy of the emitted photons fall off rapidly, and as a result maximally rotating $\mathrm{PBHs}$ probe higher mass window than their nonspinning counterparts. Because of the lower energy reach and larger effective area, AMEGO is able to probe into asteroid-mass windows compared to the previous gamma-ray observatories such as INTEGRAL [106], Fermi [107], and CRGO [108] for both nonrotating and maximally rotating PBHs. The projected upper limit from AMEGO excludes nonrotating (maximally rotating, $\left.a_{*}=0.9999\right)$ PBHs as the sole component of DM upto $7 \times 10^{17} \mathrm{~g}\left(4 \times 10^{18} \mathrm{~g}\right)$. The kinks in the exclusion limits

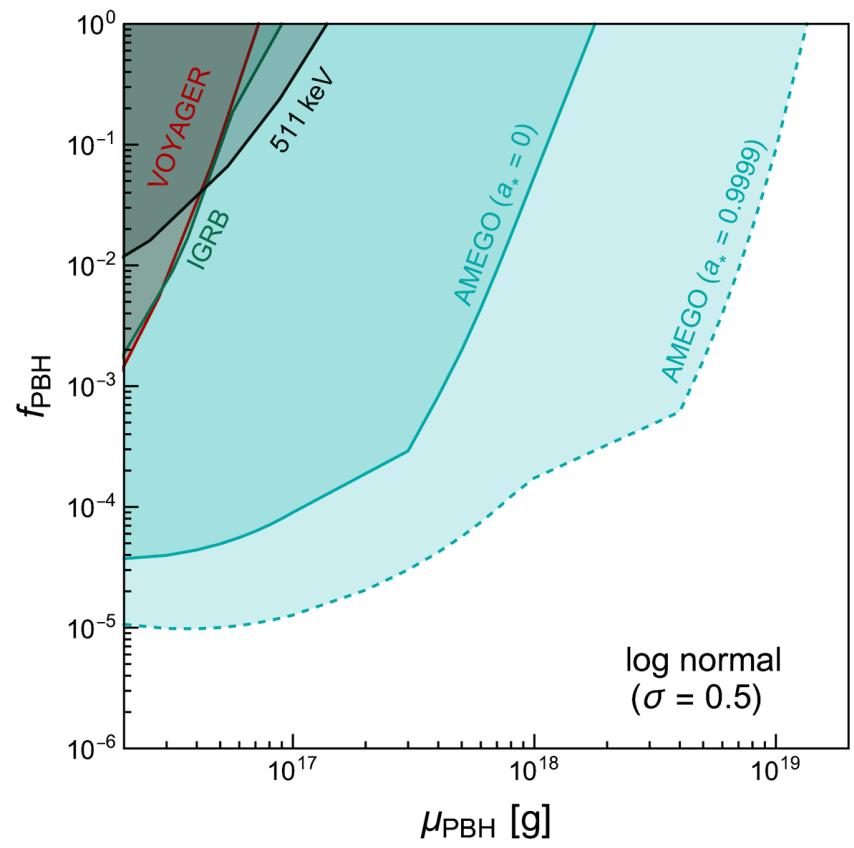

FIG. 4. Projected upper limit (95\% C.L.) on the DM fraction of PBHs, $f_{\mathrm{PBH}}$, from near future $\mathrm{MeV}$ telescope AMEGO. Lognormal mass distribution with a width $\sigma=0.5$ is considered in this plot. Results for nonrotating PBHs $\left(a_{*}=0\right)$ and maximally rotating PBHs $\left(a_{*}=0.9999\right)$ are shown by the solid line and the dashed line respectively. The constraints are derived by considering an NFW density profile of the ultralight PBHs. The existing constraints on ultralight nonspinning PBHs from Voyager-1 measurement of positron flux (shaded red) [26], extra-Galactic gamma ray emission(shaded green) $[22,29,109]$, and SPI/INTEGRAL $511 \mathrm{keV}$ emission line with $1.5 \mathrm{kpc}$ positron annihilation region and isothermal DM profile (shaded black) $[14,27,28]$ are also shown for comparison. for both nonrotating and maximally rotating $\mathrm{PBHs}$ are due to finite number of mass point samplings. Our exclusion limits start from $2 \times 10^{16} \mathrm{~g}$ as lighter PBHs mostly evaporate to higher-energy photons, outside of our considered energy range. Quantitatively, for a nonrotating $\mathrm{PBH}$ of mass $10^{16} \mathrm{~g}\left(2 \times 10^{16} \mathrm{~g}\right), \sim 30 \%(\sim 97 \%)$ of the evaporation spectrum resides in our considered energy interval, explaining the choice of $2 \times 10^{16} \mathrm{~g}$ as the minimum PBH mass for this analysis.

Fig. 4 shows the projected upper limits (95\% C.L.) on $\mathrm{DM}$ fraction of PBHs, $f_{\mathrm{PBH}}$, that can be derived from future $\mathrm{MeV}$ telescope AMEGO by assuming no signal present in the data for an extended mass distribution. Log-normal mass distribution of $\mathrm{PBHs}$, a motivated scenario from several inflationary models, with a width of $\sigma=0.5$ is considered to derive the exclusion limits. The density profile of PBHs are assumed to be NFW, however, the result degrades by at most a factor of two for cored density profiles. The solid (dashed) lines correspond to nonrotating (maximally rotating) PBHs. For this particular mass distribution, our projections exclude upto $\sim 2 \times 10^{18} \mathrm{~g}$ $\left(\sim 10^{19} \mathrm{~g}\right)$ for nonrotating (approaching maximal rotation $\left.a_{*}=0.9999\right)$ PBHs. Similar to the monochromatic mass distributions, here also, AMEGO probes better than other proposed $\mathrm{MeV}$ telescopes because of its lower energy reach and larger effective area. Similar to Fig. 3, here also, the kinks in the exclusion limits at around $3 \times 10^{17} \mathrm{~g}$ for nonrotating PBHs, and at $\sim 3 \times 10^{18} \mathrm{~g}$ for maximally rotating $\mathrm{PBHs}$ are due to the finite number of mass point samplings.

Fig. 5 shows the confidence ellipses at $68.3 \%$ C.L. and 95\% C.L. for all signal and background parameters. Nonrotating PBHs with a monochromatic mass distribution centered at $10^{17} \mathrm{~g}$ and an NFW density profile is assumed for this figure. The confidence ellipses show degeneracies among all of the parameters and the parameters of the ellipses are computed from [111]. For example, amplitude of the Galactic background $\left(A_{\mathrm{bkg}}\right)$, amplitude of the extraGalactic background $\left(A_{\mathrm{bkg}}^{\mathrm{eg}}\right)$, and the exponential cutoff energy for the Galactic background $\left(E_{\text {cut }}\right)$ are correlated with the signal parameter, DM fraction of $\mathrm{PBHs}\left(f_{\mathrm{PBH}}\right)$. However, the power law index of the Galactic background $(\alpha)$, power law index of the extra-Galactic background $\left(\alpha^{\mathrm{eg}}\right)$, and index of the exponential cut off energy in the Galactic background $(\gamma)$ are anticorrelated with $f_{\mathrm{PBH}}$. From the confidence ellipses, it is also evident that the correlation coefficient $r_{i j}\left(=\mathcal{F}_{i j}^{-1} / \sqrt{\mathcal{F}_{i i}^{-1} \mathcal{F}_{j j}^{-1}}\right)$ between $A_{\mathrm{bkg}}^{\mathrm{eg}}$ and $f_{\mathrm{PBH}}$ $(r=0.697)$ is much stronger than the correlation between $E_{\text {cut }}$ and $f_{\mathrm{PBH}}(r=0.468)$. In Fig. 5 , we also show the best fit values of all background parameters as well as their corresponding error bars by the mean and variance of the blue Gaussian curves. 


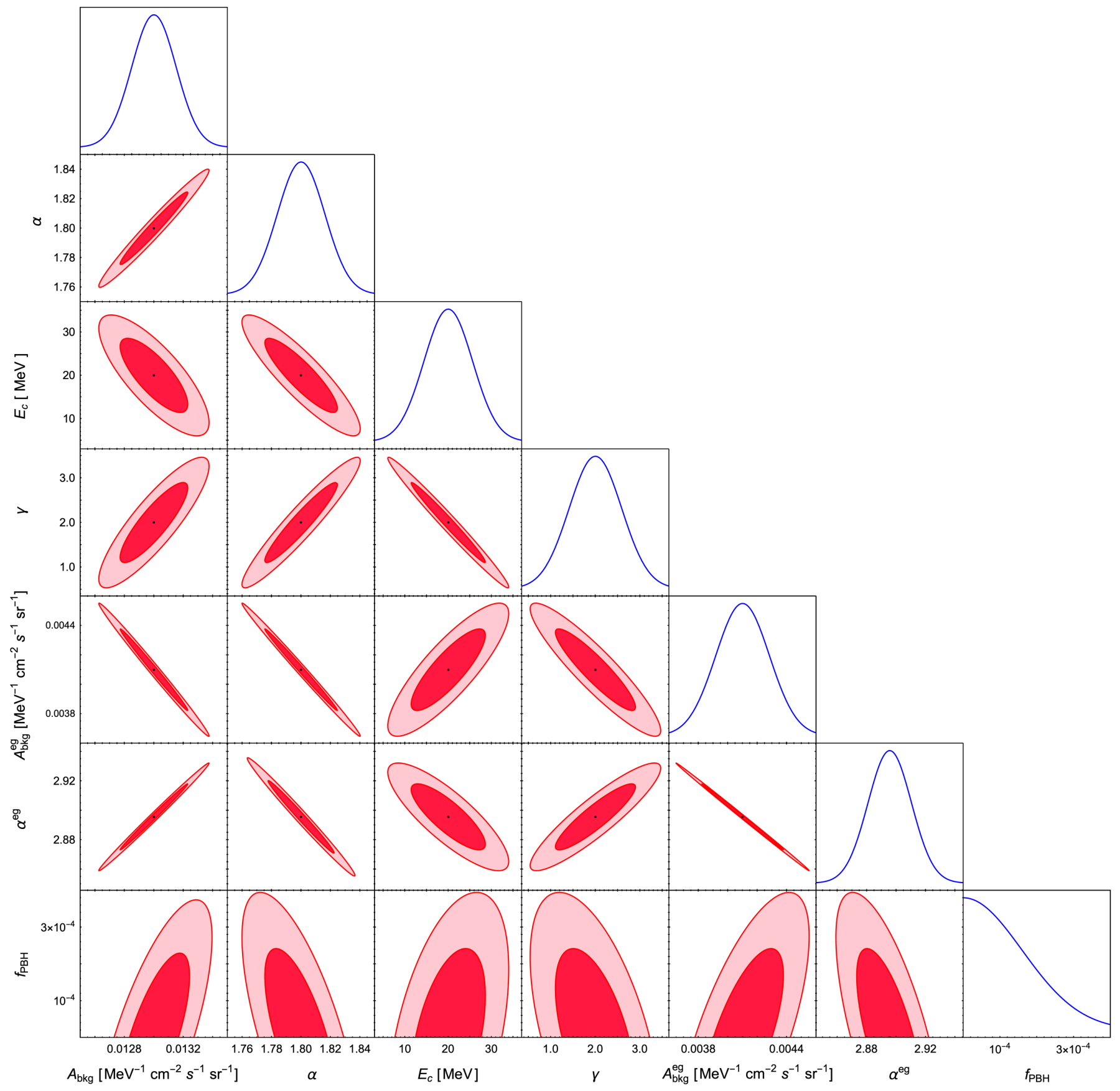

FIG. 5. Confidence ellipses at $68.3 \%$ C.L. (1- $\sigma$, dark red) and $95 \%$ C.L. (2- $\sigma$, light red) for all background and signal parameters. A larger correlation between parameters appears as a more tilted confidence ellipse. For this corner plot we have assumed nonrotating and uncharged PBHs with a monochromatic mass distribution centered at $10^{17} \mathrm{~g}$, following a NFW density profile. Black dots represent bestfit (i.e., our chosen fiducial) values of the background parameters, and we assume a fiducial $f_{\mathrm{PBH}}=0$. The predicted posteriors for all parameters are shown by the Gaussian curves in blue.

\section{SUMMARY AND CONCLUSIONS}

PBHs in the asteroid-mass range, $\sim 10^{17}-10^{23} \mathrm{~g}$, can make up the entire DM density and it is very important to conclusively probe these candidates. We propose a strategy to decisively probe a part of this parameter space. At the lower end of this mass range, PBHs with masses $\sim 10^{17} \mathrm{~g}-10^{18} \mathrm{~g}$ have Hawking temperatures in the range of $0.01 \mathrm{MeV}$ to $0.1 \mathrm{MeV}$, implying that substantial evaporated photons are produced by them around these energy scales. Near-future soft gamma-ray telescopes like AMEGO, with its large effective area and improved background rejection capabilities, can search for these photons and investigate this hard to probe parameter space. The most efficient search strategy involves observations of 
the region around the Galactic Center. We include the Galactic astrophysical background produced by cosmic rays and the measured extra-galactic gamma-ray background in our projected search strategy. Our projections show that AMEGO can exclude nonrotating PBHs as the sole component of DM up to $\sim 7 \times 10^{17} \mathrm{~g}$. We demonstrate that maximal rotation as well as extended mass distribution of the PBHs allow us to explore larger ranges of $\mathrm{PBH}$ masses. We also predict that the projected exclusions on PBH DM in the mass range $\sim 10^{16}-10^{17} \mathrm{~g}$ will be much stronger than the existing limits. The projections presented in this work are robust to the different choices of DM density profiles. At higher $\mathrm{PBH}$ masses in this range, the
Hawking radiation flux gets smaller and thus much larger instruments need to be built in order to detect the evaporation signature. In the absence of much larger telescopes, other techniques need to be developed in order to probe the complete parameter space of asteroid-mass PBHs.

\section{ACKNOWLEDGMENTS}

We thank Basudeb Dasgupta and Thomas Edwards for discussions and useful suggestions. We especially thank Tracy R. Slatyer for early discussions which led to this work. J. B. M. is supported by a Clay Fellowship at the Smithsonian Astrophysical Observatory.
[1] N. Aghanim et al. (Planck Collaboration), Planck 2018 results. VI. Cosmological parameters, Astron. Astrophys. 641, A6 (2020).

[2] K. Pardo and D. N. Spergel, What is the Price of Abandoning Dark Matter? Cosmological Constraints on Alternative Gravity Theories, Phys. Rev. Lett. 125, 211101 (2020).

[3] L. E. Strigari, Galactic searches for dark matter, Phys. Rep. 531, 1 (2013).

[4] T. R. Slatyer, Indirect detection of dark matter, in Theoretical Advanced Study Institute in Elementary Particle Physics: Anticipating the Next Discoveries in Particle Physics (World Scientific, Singapore, 2018), pp. 297353, https://doi.org/10.1142/9789813233348_0005.

[5] T. Lin, Dark matter models and direct detection, Proc. Sci., 333 (2019) 009 [arXiv:1904.07915].

[6] A. Boveia and C. Doglioni, Dark matter searches at colliders, Annu. Rev. Nucl. Part. Sci. 68, 429 (2018).

[7] Y. B. Zel'dovich and I. D. Novikov, The hypothesis of cores retarded during expansion and the hot cosmological model, Astron. Zh. 43, 758 (1966), http://cdsads.u-strasbg .fr/pdf/1967SvA....10..602Z.

[8] S. Hawking, Gravitationally collapsed objects of very low mass, Mon. Not. R. Astron. Soc. 152, 75 (1971).

[9] B. J. Carr and S. W. Hawking, Black holes in the early Universe, Mon. Not. R. Astron. Soc. 168, 399 (1974).

[10] G. F. Chapline, Cosmological effects of primordial black holes, Nature (London) 253, 251 (1975).

[11] A. Katz, J. Kopp, S. Sibiryakov, and W. Xue, Femtolensing by dark matter revisited, J. Cosmol. Astropart. Phys. 12 (2018) 005.

[12] P. Montero-Camacho, X. Fang, G. Vasquez, M. Silva, and C. M. Hirata, Revisiting constraints on asteroid-mass primordial black holes as dark matter candidates, J. Cosmol. Astropart. Phys. 08 (2019) 031.

[13] N. Smyth, S. Profumo, S. English, T. Jeltema, K. McKinnon, and P. Guhathakurta, Updated constraints on asteroidmass primordial black holes as dark matter, Phys. Rev. D 101, 063005 (2020).
[14] B. Dasgupta, R. Laha, and A. Ray, Neutrino and Positron Constraints on Spinning Primordial Black Hole Dark Matter, Phys. Rev. Lett. 125, 101101 (2020).

[15] B. Carr, K. Kohri, Y. Sendouda, and J. Yokoyama, Constraints on primordial black holes, arXiv:2002.12778.

[16] A. M. Green and B. J. Kavanagh, Primordial Black Holes as a dark matter candidate, J. Phys. G 48, 043001 (2021).

[17] B. Carr and F. Kuhnel, Primordial black holes as dark matter: Recent developments, Annu. Rev. Nucl. Part. Sci. 70, 355 (2020).

[18] B. P. Abbott et al. (LIGO Scientific, Virgo Collaborations), Observation of Gravitational Waves from a Binary Black Hole Merger, Phys. Rev. Lett. 116, 061102 (2016).

[19] S. Bird, I. Cholis, J. B. Muñoz, Y. Ali-Haimoud, M. Kamionkowski, E. D. Kovetz, A. Raccanelli, and A. G. Riess, Did LIGO Detect Dark Matter?, Phys. Rev. Lett. 116, 201301 (2016).

[20] S. Clesse and J. Garcia-Bellido, The clustering of massive Primordial Black Holes as Dark Matter: Measuring their mass distribution with Advanced LIGO, Phys. Dark Universe 15, 142 (2017).

[21] M. Sasaki, T. Suyama, T. Tanaka, and S. Yokoyama, Primordial Black Hole Scenario for the GravitationalWave Event GW150914, Phys. Rev. Lett. 117, 061101 (2016).

[22] A. Arbey, J. Auffinger, and J. Silk, Constraining primordial black hole masses with the isotropic gamma ray background, Phys. Rev. D 101, 023010 (2020).

[23] S. Clark, B. Dutta, Y. Gao, L. E. Strigari, and S. Watson, Planck constraint on relic primordial black holes, Phys. Rev. D 95, 083006 (2017).

[24] S. Wang, Y.-F. Wang, Q.-G. Huang, and T. G. F. Li, Constraints on the Primordial Black Hole Abundance from the First Advanced LIGO Observation Run Using the Stochastic Gravitational-Wave Background, Phys. Rev. Lett. 120, 191102 (2018).

[25] H. Poulter, Y. Ali-Haimoud, J. Hamann, M. White, and A. G. Williams, CMB constraints on ultra-light primordial black holes with extended mass distributions, arXiv:1907.06485. 
[26] M. Boudaud and M. Cirelli, Voyager $1 e^{ \pm}$Further Constrain Primordial Black Holes as Dark Matter, Phys. Rev. Lett. 122, 041104 (2019).

[27] W. DeRocco and P. W. Graham, Constraining Primordial Black Hole Abundance with the Galactic $511 \mathrm{keV}$ Line, Phys. Rev. Lett. 123, 251102 (2019).

[28] R. Laha, Primordial Black Holes as a Dark Matter Candidate Are Severely Constrained by the Galactic Center $511 \mathrm{keV} \gamma$-Ray Line, Phys. Rev. Lett. 123, 251101 (2019).

[29] G. Ballesteros, J. Coronado-Blázquez, and D. Gaggero, $\mathrm{X}$-ray and gamma-ray limits on the primordial black hole abundance from Hawking radiation, Phys. Lett. B 808, 135624 (2020).

[30] R. Laha, J. B. Munoz, and T. R. Slatyer, INTEGRAL constraints on primordial black holes and particle dark matter, Phys. Rev. D 101, 123514 (2020).

[31] R. Allsman et al. (Macho Collaboration), MACHO project limits on black hole dark matter in the 1-30 solar mass range, Astrophys. J. Lett. 550, L169 (2001).

[32] P. Tisserand et al. (EROS-2 Collaboration), , Limits on the macho content of the galactic Halo from the EROS-2 survey of the magellanic clouds, Astron. Astrophys. 469, 387 (2007).

[33] H. Niikura, M. Takada, S. Yokoyama, T. Sumim, and S. Masaki, Constraints on Earth-mass primordial black holes from OGLE 5-year microlensing events, Phys. Rev. D 99, 083503 (2019).

[34] M. Oguri, J. M. Diego, N. Kaiser, P. L. Kelly, and T. Broadhurst, Understanding caustic crossings in giant arcs: Characteristic scales, event rates, and constraints on compact dark matter, Phys. Rev. D 97, 023518 (2018).

[35] M. Zumalacarregui and U. Seljak, Limits on Stellar-Mass Compact Objects as Dark Matter from Gravitational Lensing of Type Ia Supernovae, Phys. Rev. Lett. 121, 141101 (2018).

[36] B. Abbott et al. (LIGO Scientific, Virgo Collaborations), Search for Subsolar Mass Ultracompact Binaries in Advanced LIGO's Second Observing Run, Phys. Rev. Lett. 123, 161102 (2019).

[37] B. J. Kavanagh, D. Gaggero, and G. Bertone, Merger rate of a subdominant population of primordial black holes, Phys. Rev. D 98, 023536 (2018).

[38] T. D. Brandt, Constraints on MACHO dark matter from compact stellar systems in ultra-faint dwarf galaxies, Astrophys. J. Lett. 824, L31 (2016).

[39] S. M. Koushiappas and A. Loeb, Dynamics of Dwarf Galaxies Disfavor Stellar-Mass Black Holes as Dark Matter, Phys. Rev. Lett. 119, 041102 (2017).

[40] M. A. Monroy-Rodriguez and C. Allen, The end of the MACHO era- revisited: New limits on MACHO masses from halo wide binaries, Astrophys. J. 790, 159 (2014).

[41] P. D. Serpico, V. Poulin, D. Inman, and K. Kohri, Cosmic microwave background bounds on primordial black holes including dark matter Halo accretion, Phys. Rev. Research 2, 023204 (2020).

[42] A. Hektor, G. Hutsi, L. Marzola, M. Raidal, V. Vaskonen, and H. Veermae, Constraining primordial black holes with the EDGES 21-cm absorption signal, Phys. Rev. D 98, 023503 (2018).
[43] J. Manshanden, D. Gaggero, G. Bertone, R. M. Connors, and M. Ricotti, Multi-wavelength astronomical searches for primordial black holes, J. Cosmol. Astropart. Phys. 06 (2019) 026.

[44] A. Hektor, G. Hutsi, and M. Raidal, Constraints on primordial black hole dark matter from Galactic center X-ray observations, Astron. Astrophys. 618, A139 (2018).

[45] M. Raidal, V. Vaskonen, and H. Veermäe, Gravitational waves from primordial black hole mergers, J. Cosmol. Astropart. Phys. 09 (2017) 037.

[46] M. W. Sammons, J.-P. Macquart, R. D. Ekers, R. M. Shannon, H. Cho, J. X. Prochaska, A. T. Deller, and C. K. Day, First constraints on compact dark matter from fast radio burst microstructure, Astrophys. J. 900, 122 (2020).

[47] P. Lu, V. Takhistov, G. B. Gelmini, K. Hayashi, Y. Inoue, and A. Kusenko, Constraining primordial black holes with dwarf galaxy heating, Astrophys. J. Lett. 908, L23 (2021).

[48] A. H. Nitz and Y.-F. Wang, Search for Gravitational Waves from High-Mass-Ratio Compact-Binary Mergers of Stellar Mass and Subsolar Mass Black Holes, Phys. Rev. Lett. 126, 021103 (2021).

[49] A. H. Nitz and Y.-F. Wang, Search for gravitational waves from the coalescence of sub-solar mass and eccentric compact binaries, arXiv:2102.00868.

[50] R. Laha, P. Lu, and V. Takhistov, Gas heating from spinning and nonspinning evaporating primordial black holes, arXiv:2009.11837.

[51] H. Kim, A constraint on light primordial black holes from the interstellar medium temperature, arXiv:2007.07739.

[52] M. H. Chan and C. M. Lee, Constraining primordial black hole fraction at the galactic centre using radio observational data, Mon. Not. R. Astron. Soc. 497, 1212 (2020).

[53] A. Dolgov, A. Kuranov, N. Mitichkin, S. Porey, K. Postnov, O. Sazhina, and I. V. Simkin, On mass distribution of coalescing black holes, J. Cosmol. Astropart. Phys. 12 (2020) 017.

[54] K. W. K. Wong, G. Franciolini, V. De Luca, V. Baibhav, E. Berti, P. Pani, and A. Riotto, Constraining the primordial black hole scenario with Bayesian inference and machine learning: The GWTC-2 gravitational wave catalog, Phys. Rev. D 103, 023026 (2021).

[55] G. Hütsi, M. Raidal, V. Vaskonen, and H. Veermäe, Two populations of LIGO-Virgo black holes, J. Cosmol. Astropart. Phys. 03 (2021) 068.

[56] A. Coogan, L. Morrison, and S. Profumo, Direct Detection of Hawking Radiation from Asteroid-Mass Primordial Black Holes, Phys. Rev. Lett. 126, 171101 (2021).

[57] A. Halder and M. Pandey, Investigating the effect of $\mathrm{PBH}$, dark matter-baryon and dark matter-dark energy interaction on EDGES in $21 \mathrm{~cm}$ signal, arXiv:2101.05228.

[58] J. B. Munoz, E. D. Kovetz, L. Dai, and M. Kamionkowski, Lensing of Fast Radio Bursts as a Probe of Compact Dark Matter, Phys. Rev. Lett. 117, 091301 (2016).

[59] R. Laha, Lensing of fast radio bursts: Future constraints on primordial black hole density with an extended mass function and a new probe of exotic compact fermion and boson stars, Phys. Rev. D 102, 023016 (2020).

[60] A. Katz, J. Kopp, S. Sibiryakov, and W. Xue, Looking for MACHOs in the spectra of fast radio bursts, Mon. Not. R. Astron. Soc. 496, 564 (2020). 
[61] S. Jung and C. S. Shin, Gravitational-Wave Fringes at LIGO: Detecting Compact Dark Matter by Gravitational Lensing, Phys. Rev. Lett. 122, 041103 (2019).

[62] F. Kuhnel, A. Matas, G. D. Starkman, and K. Freese, Waves from the Centre: Probing PBH and other macroscopic dark matter with LISA, Eur. Phys. J. C 80, 627 (2020).

[63] R.-g. Cai, S. Pi, and M. Sasaki, Gravitational Waves Induced by non-Gaussian Scalar Perturbations, Phys. Rev. Lett. 122, 201101 (2019).

[64] S. Jung and T. Kim, Gamma-ray burst lensing parallax: Closing the primordial black hole dark matter mass window, Phys. Rev. Research 2, 013113 (2020).

[65] Y. Bai and N. Orlofsky, Microlensing of X-ray pulsars: A method to detect primordial black hole dark matter, Phys. Rev. D 99, 123019 (2019).

[66] Y.-F. Wang, Q.-G. Huang, T. G. Li, and S. Liao, Searching for primordial black holes with stochastic gravitationalwave background in the space-based detector frequency band, Phys. Rev. D 101, 063019 (2020).

[67] J. A. Dror, H. Ramani, T. Trickle, and K. M. Zurek, Pulsar timing probes of primordial black holes and subhalos, Phys. Rev. D 100, 023003 (2019).

[68] H.-K. Guo, J. Shu, and Y. Zhao, Using LISA-like gravitational wave detectors to search for primordial black holes, Phys. Rev. D 99, 023001 (2019).

[69] B. Dutta, A. Kar, and L. E. Strigari, Constraints on MeV dark matter and primordial black holes: Inverse Compton signals at the SKA, J. Cosmol. Astropart. Phys. 03 (2021) 011.

[70] A. Kusenko, M. Sasaki, S. Sugiyama, M. Takada, V. Takhistov, and E. Vitagliano, Exploring Primordial Black Holes from the Multiverse with Optical Telescopes, Phys. Rev. Lett. 125, 181304 (2020).

[71] S. Sugiyama, V. Takhistov, E. Vitagliano, A. Kusenko, M. Sasaki, and M. Takada, Testing stochastic gravitational wave signals from primordial black holes with optical telescopes, Phys. Lett. B 814, 136097 (2021).

[72] N. Bhaumik and R. K. Jain, Stochastic induced gravitational waves and lowest mass limit of primordial black holes with the effects of reheating, arXiv:2009.10424.

[73] D. N. Page, Particle emission rates from a black hole: Massless particles from an uncharged, nonrotating hole, Phys. Rev. D 13, 198 (1976).

[74] D. N. Page, Particle emission rates from a black hole. 2. Massless particles from a rotating hole, Phys. Rev. D 14, 3260 (1976).

[75] J. H. MacGibbon, B. J. Carr, and D. N. Page, Do evaporating black holes form photospheres?, Phys. Rev. D 78, 064043 (2008).

[76] P. N. Okele and M. J. Rees, Observational consequences of positron production by evaporating black holes, Astron. Astrophys. 81, 263 (1980), http://articles.adsabs.harvard .edu/pdf/1980A\%26A....81..2630.

[77] P. Okeke, The primary source and the fates of galactic positrons, Astrophys. Space Sci. 71, 371 (1980).

[78] J. H. MacGibbon and B. J. Carr, Cosmic rays from primordial black holes, Astrophys. J. 371, 447 (1991).

[79] C. Bambi, A. D. Dolgov, and A. A. Petrov, Primordial black holes and the observed Galactic 511-keV line, Phys. Lett. B 670, 174 (2008).
[80] P. Stöcker, M. Krämer, J. Lesgourgues, and V. Poulin, Exotic energy injection with ExoCLASS: Application to the Higgs portal model and evaporating black holes, J. Cosmol. Astropart. Phys. 03 (2018) 018.

[81] S. K. Acharya and R. Khatri, CMB and BBN constraints on evaporating primordial black holes revisited, J. Cosmol. Astropart. Phys. 06 (2020) 018.

[82] C. Keith, D. Hooper, N. Blinov, and S. D. McDermott, Constraints on primordial black holes from big bang nucleosynthesis revisited, Phys. Rev. D 102, 103512 (2020).

[83] https://nssdc.gsfc.nasa.gov/planetary/factsheet/asteroidfact .html

[84] C. Kouvaris, P. Tinyakov, and M. H. Tytgat, NonPrimordial Solar Mass Black Holes, Phys. Rev. Lett. 121, 221102 (2018).

[85] B. Dasgupta, R. Laha, and A. Ray, Low Mass Black Holes from Dark Core Collapse, Phys. Rev. Lett. 126, 141105 (2021).

[86] S. Shandera, D. Jeong, and H. S. G. Gebhardt, Gravitational Waves from Binary Mergers of Subsolar Mass Dark Black Holes, Phys. Rev. Lett. 120, 241102 (2018).

[87] J. McEnery, A. van der Horst, A. Dominguez, A. Moiseev, A. Marcowith, A. Harding et al., All-sky medium energy gamma-ray observatory: Exploring the extreme multimessenger universe, Bull. Am. Astron. Soc. 51, 245 (2019), arXiv:1907.07558.

[88] J. H. MacGibbon and B. R. Webber, Quark and gluon jet emission from primordial black holes: The instantaneous spectra, Phys. Rev. D 41, 3052 (1990).

[89] J.H. MacGibbon, Quark and gluon jet emission from primordial black holes. 2. The Lifetime emission, Phys. Rev. D 44, 376 (1991).

[90] A. Arbey and J. Auffinger, BlackHawk: A public code for calculating the Hawking evaporation spectra of any black hole distribution, Eur. Phys. J. C 79, 693 (2019).

[91] R. Bartels, D. Gaggero, and C. Weniger, Prospects for indirect dark matter searches with $\mathrm{MeV}$ photons, J. Cosmol. Astropart. Phys. 05 (2017) 001.

[92] A. W. Strong, K. Bennett, H. Bloemen, R. Diehl, W. Hermsen, D. Morris et al., Diffuse continuum gamma rays from the Galaxy observed by COMPTEL, Astron. Astrophys. 292, 82 (1994), http://cdsads.u-strasbg.fr/pdf/ 1994A\%26A...292...82S.

[93] A. Strong, H. Bloemen, R. Diehl, W. Hermsen, and V. Schoenfelder, Comptel skymapping: A New approach using parallel computing, Astrophys. Lett. Commun. 39, 209 (1999), http://cdsads.u-strasbg.fr/pdf/1999ApL\%26C. .39..209S.

[94] A. Strong (Fermi-LAT Collaboration), Interstellar gamma rays and cosmic rays: New insights from Fermi-LAT AND INTEGRAL, in ICATPP Conference on Cosmic Rays for Particle and Astroparticle Physics (World Scientific, Singapore, 2011), pp. 473-481,https://doi.org/10.1142/ 9789814329033_0059.

[95] K. Watanabe, D. H. Hartmann, M. D. Leising, L. S. The, G. H. Share, and R. L. Kinzer, The cosmic $\gamma$-ray background from supernovae, in Proceedings of the Fourth Compton Symposium, edited by C. D. Dermer, M. S. Strickman, and J. D. Kurfess, Vol. 410 of American Institute of Physics 
Conference Series (AIP Publishing LLC, 1997), pp. 12231227,https://doi.org/10.1063/1.53933.

[96] Y. Fukada, S. Hayakawa, M. Ikeda, I. Kasahara, F. Makino, and Y. Tanaka, Rocket observation of energy spectrum of diffuse hard X-rays, Astrophys. Space Sci. 32, L1 (1975).

[97] D. Gruber, J. Matteson, L. Peterson, and G. Jung, The spectrum of diffuse cosmic hard x-rays measured with heao-1, Astrophys. J. 520, 124 (1999).

[98] R. L. Kinzer, G. V. Jung, D. E. Gruber, J. L. Matteson, and L.E. Peterson, Diffuse cosmic gamma radiation measured by HEAO 1, Astrophys. J. 475, 361 (1997).

[99] G. Weidenspointner et al., The comptel instrumental line background, AIP Conf. Proc. 510, 581 (2000).

[100] T. D. P. Edwards and C. Weniger, A fresh approach to forecasting in astroparticle physics and dark matter searches, J. Cosmol. Astropart. Phys. 02 (2018) 021.

[101] T. D. P. Edwards and C. Weniger, swordfish: Efficient forecasting of new physics searches without Monte Carlo, arXiv:1712.05401.

[102] M. T. Doornhein, Uses and limitations of fisher forecasting in setting upper limits on the interaction strength of dark matter, MSc thesis, 2018, https://scripties.uba.uva.nl/ search?id=650205.

[103] https://asd.gsfc.nasa.gov/amego/technical.html
[104] J. F. Navarro, C. S. Frenk, and S. D. M. White, A Universal density profile from hierarchical clustering, Astrophys. J. 490, 493 (1997).

[105] K. C. Y. Ng, R. Laha, S. Campbell, S. Horiuchi, B. Dasgupta, K. Murase, and J.F. Beacom, Resolving small-scale dark matter structures using multisource indirect detection, Phys. Rev. D 89, 083001 (2014).

[106] C. Winkler et al., The INTEGRAL mission, Astron. Astrophys. 411, L1 (2003).

[107] W. B. Atwood et al. (Fermi-LAT Collaboration), The large area telescope on the fermi gamma-ray space telescope mission, Astrophys. J. 697, 1071 (2009).

[108] V. Schoenfelder, H. Aarts, K. Bennett, H. de Boer, J. Clear, W. Collmar et al., Instrument description and performance of the imaging gamma-ray telescope COMPTEL aboard the compton gamma-ray observatory, Astrophys. J. Suppl. 86, 657 (1993), http://cdsads.u-strasbg.fr/pdf/1993ApJS.. $.86 . .657 \mathrm{~S}$.

[109] B. Carr, K. Kohri, Y. Sendouda, and J. Yokoyama, New cosmological constraints on primordial black holes, Phys. Rev. D 81, 104019 (2010).

[110] H. Niikura et al., Microlensing constraints on primordial black holes with Subaru/HSC Andromeda observations, Nat. Astron. 3, 524 (2019).

[111] D. Coe, Fisher matrices and confidence ellipses: A quickstart guide and software, arXiv:0906.4123. 